Décadrages Décadrages

cınéma, à travers champs Cinéma, à travers champs

6 | 2005

Train et cinéma

\title{
Train, cinéma et modernité : entre hystérie et hypnose
}

Mireille Berton

\section{(2) OpenEdition}

Journals

\section{Édition électronique}

URL : http://journals.openedition.org/decadrages/476

DOI : $10.4000 /$ decadrages.476

ISSN : 2297-5977

Éditeur

Association Décadrages

Édition imprimée

Date de publication : 10 octobre 2005

Pagination : 8-21

ISBN : 978-2-9700582-2-9

ISSN : 2235-7823

Référence électronique

Mireille Berton, «Train, cinéma et modernité : entre hystérie et hypnose », Décadrages [En ligne],

6 | 2005, mis en ligne le 20 janvier 2014, consulté le 03 mai 2019. URL : http://

journals.openedition.org/decadrages/476 ; DOI : 10.4000/decadrages.476

(B) Décadrages 


\section{Train, cinéma et modernité : entre hystérie}

\section{et hypnose}

par Mireille Berton

1 Voir à ce sujet l'ouvrage de Lynne Kirby qui propose une excellente synthèse des liens entre train et cinéma: Parallel Tracks: the Railroad and Silent Cinema, University of Exeter Press, Exeter, 1997. Voir aussi Livio Belloï, Le regard retourné. Aspects du cinéma des premiers temps, Nota bene/Méridiens Klincksieck, Québec/Paris, 2002; Marc-Emmanuel Mélon, "Le voyage en train et en images: une expérience photographique de la discontinuité et de la fragmentation", dans François Albera, Marta Braun et André Gaudreault (éd.), Arrêt sur image, fragmentation du temps, Ed. Payot (Cinéma), Lausanne, 2002, pp. 47-68; Jacques Aumont, L'œil interminable. Cinéma et peinture, Séguier, Paris, 1995, pp. 43-45.

2 Article anonyme d'un journaliste ayant assisté à la première projection des films Lumière le 28 décembre 1895. "Le Cinématographe: une merveille photographique", Le Radical, 30 décembre 1895, dans Georges Sadoul, Lumière et Méliès, Lherminier, Paris, 1985, p. 101.

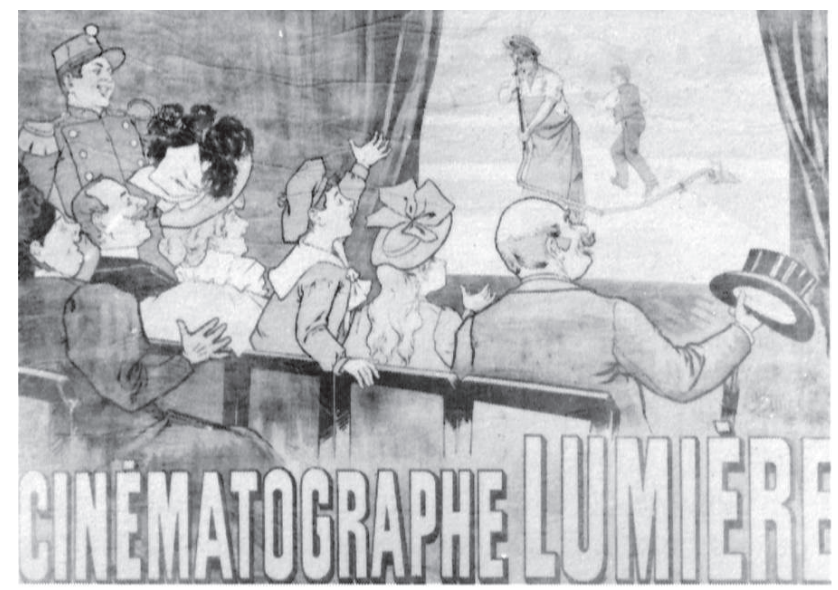

Nouvelles machines de voyage et de vision nées au XIX ${ }^{\mathrm{e}}$ siècle, le train et le cinéma ont fréquemment été étudiés sous l'angle de leurs affinités multiples, que ce soit au niveau historique, technologique, social, culturel ou esthétique $\mathbf{1}$. Les auteurs ont notamment démontré le rôle crucial joué par le chemin de fer dans la construction d'un paradigme perceptif fondé sur l'appréhension d'une série d'images délimitées par un cadre et défilant rapidement devant un voyageur-spectateur immobile, paradigme que le cinéma absorbera quelques décennies plus tard pour offrir à un public déjà familier des spectacles de lanterne magique, une expérience cénesthésique dotée du pouvoir de le transporter artificiellement vers un ailleurs, "avec toute l'illusion de la vie réelle» $\mathbf{2}$. Si la très forte impression de réalité produite par le Cinématographe constitue l'une des composantes majeures de sa réception critique, un autre aspect attire l'attention des premiers spectateurs qui soulignent la gêne, plus ou moins marquée, face au très fort clignotement de l'image. Georges Méliès, qui 
assiste à la projection des films Lumière à Paris en décembre 1895, se plaint d'une vibration fastidieuse qui oblige le public à se frotter les yeux $^{3}$, geste qui pourrait métaphoriser la dimension ambivalente d'un média qui à la fois envoûte - on n'en croit pas ses yeux - et irrite - on se soulage d'un léger malaise oculaire.

Sur le plan psychophysiologique donc, le cinéma semble d'emblée provoquer deux effets contradictoires puisque, d'un côté, il happe littéralement le sujet percevant dans une représentation plus vraie que nature, annihilant ainsi toute conscience proprioceptive, de l'autre il génère une fatigue visuelle due, notamment, à une fréquence de fusion insuffisante pour éviter le scintillement de l'image4. Oscillant entre oubli de soi et hypersensibilité, le spectateur de cinéma apparaît comme le lieu où viennent se rejouer les sensations vécues par les voyageurs de chemin de fer placés dans un wagon susceptible autant de les bercer que de les bousculer, voire de les traumatiser à l'occasion d'une collision. En intégrant des machineries dont le fonctionnement dépend d'une série de mouvements à la fois continus et discontinus, intégrateurs et disloquants, ensorcelants et déstabilisants, le spectateur et le voyageur revêtent toutes les caractéristiques d'une subjectivité façonnée par la modernité. En effet, les conséquences d'une accélération inédite de l'industrialisation, de l'urbanisation, de la consommation, de la mécanisation des loisirs, ainsi que de la circulation des corps, des produits et des informations, obligent l'individu, et tout particulièrement l'habitant des grandes villes, à accommoder son système perceptif à un environnement spatio-temporel complexe. La prolifération d'images, de sons, de lumières, de mouvements et d'interactions en tout genre débouche, dès lors, sur la constitution d'un sujet dont les sens et les réflexes ont été affûtés afin de mieux répondre aux chocs physiques et psychiques en provenance du monde extérieur 5 .

Très souvent conceptualisé par les théories sociales en termes de réceptivité et de réactivité accrues ${ }^{6}$, le sujet moderne va aussi faire l'objet d'un discours médical qui tente d'élucider les causes de nouvelles pathologies se développant avec une ampleur notable à la fin du XIX siècle. La neurasthénie et l'hystérie figurent au premier rang des maladies atteignant une population urbaine particulièrement exposée aux affres de la vie moderne, et dont les suites seront appréhendées de manière souvent mitigée par les spécialistes. Or, la mise en parallèle des histoires émergeantes du train et du cinéma s'avère particulièrement féconde si on la situe en regard des psychopathologies typiques de la modernité et des thèses auxquelles elles ont donné naissance. Il s'agira donc d'évoquer ici quelques aspects participant à la genèse du sujet moderne, et ceci à
3 Cité dans Gian Piero Brunetta, Buio in sala. Cent'anni di passioni dello spettatore cinematografico, Venezia, Marsilio, 2001 [1989], p. 8.

4 La fréquence critique de fusion (appelée aussi effet-phi) consiste en un phénomène stroboscopique qui permet de synthétiser les images successives en un flux continu. Alors qu'aujourd'hui elle est fixée sur vingt-quatre images par seconde, les premiers projecteurs étaient réglés sur dix-huit images par seconde, induisant la gêne ressentie face aux tressautements du film.

5 Sur ce point, voir Mary Ann Doane, The Emergence of Cinematic Time: Modernity, Contigency, the Archive, Harward University Press, Cambridge-Londres, 2002; Leo Charney et Vanessa R. Schwartz, Cinema and the Invention of Modern Life, University of California Press, Berkeley, 1995; Jonathan Crary, L'art de l'observateur. Vision et modernité au XIXe siècle, Ed. Jacqueline Chambon, Paris, 1994 [1990].

6 Je pense notamment à la tradition ouverte par Georg Simmel dont les analyses ont été retravaillées par Siegfried Kracauer et Walter Benjamin. Voir Georg Simmel, "The Metropolis and Mental Life" [1903], The Sociology of Georg Simmel, trad. et éd. de Kurt H. Wolff, The Free Press/Collier-Macmillan Limited, New York/ Londres, 1964, pp. 409-424.

BOSTON, 1904

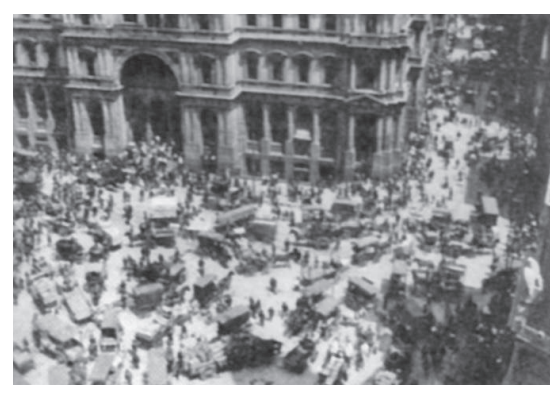




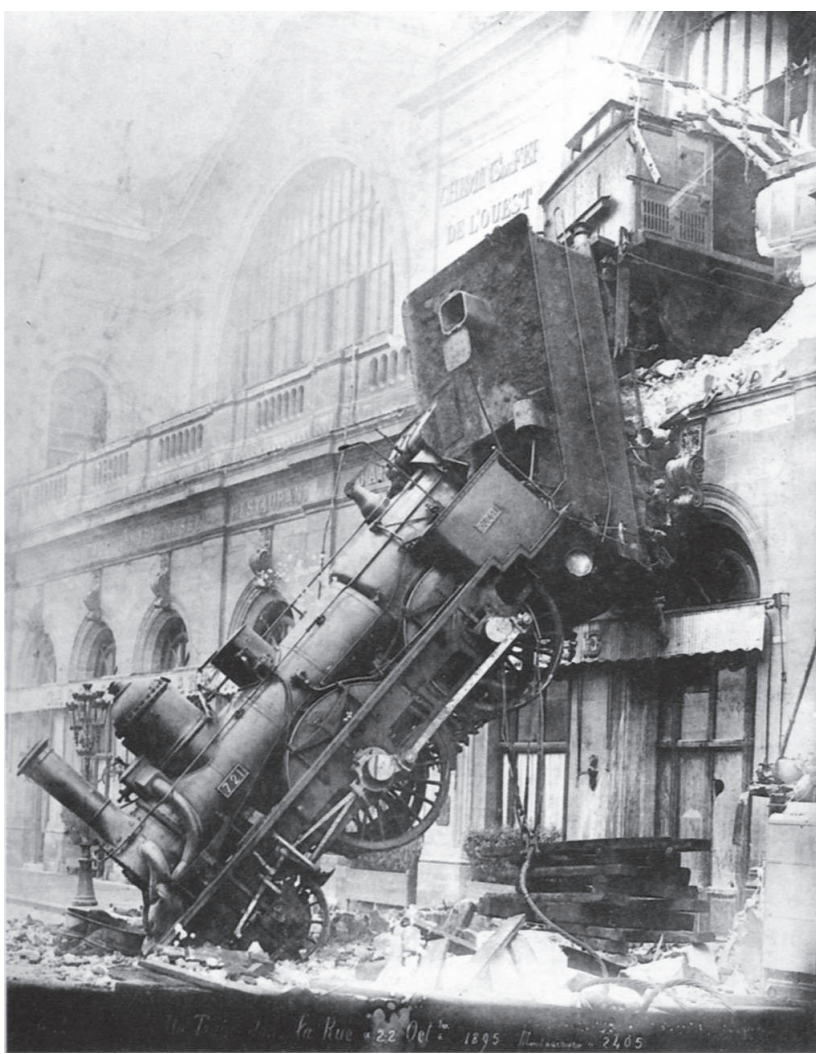

travers trois ensembles discursifs ayant tenté de définir, au tournant du $\mathrm{XX}^{\mathrm{e}}$ siècle, la spécificité d'états psychophysiologiques assumés respectivement par le voyageur, le spectateur et le névrosé dans sa variante aussi bien neurasthénique qu'hystérique.

\section{Les pathologies du chemin de fer. La neurasthénie}

Dans un passage de L'inconnu et les problèmes psychiques (1900) consacré à la «myopie intellectuelle» de certains savants, l'astronome Camille Flammarion rapporte une anecdote qui en dit long sur les peurs et les résistances suscitées par le chemin de fer dans les années 1830-1840:

«En Bavière, le collège royal de Médecine consulté déclara que les chemins de fer causeraient, s'ils étaient réalisés, le plus grand tort à la santé publique, parce qu'un mouvement aussi rapide provoquerait des ébranlements cérébraux chez les voyageurs et des vertiges dans le public extérieur, et recommanda d'enfermer les voies entre deux 
Si ce récit a pour fonction première de fustiger les préjugés de ses pairs hésitant à adhérer pleinement aux dogmes positivistes du progrès technologique et social, il évoque également les interrogations afférentes à une machine qui fait exploser les sens en raison de sa grande vitesse de déplacement. Wolfgang Schivelbusch, qui fournit une analyse précise de ces modifications de l'appareil perceptif du voyageur, précise que «la difficulté à distinguer quoi que ce soit dans le paysage traversé, en dehors des formes les plus grossières, est mentionnée dans toutes les premières descriptions de voyages en chemin de fer»8. Pris dans un flux accéléré d'images insaisissables et floues, le sujet envahi par un tel excès de stimuli encourt des lésions fréquemment signalées dans la presse spécialisée. Un article du journal médical From the Lancet explique en 1862 :

"La vitesse et la diversité des impressions fatiguent nécessairement l'œil comme le cerveau. La distance des objets qui se transforme sans cesse exige un continuel travail d'adaptation de l'appareil oculaire à travers lequel ils s'impriment sur la rétine; et la fatigue intellectuelle du cerveau à les recevoir est à peine moindre, même si elle est inconsciente; car aucun fait n'est moins controversé dans le domaine de la physiologie que celui d'après lequel une activité fonctionnelle excessive entraîne toujours un dommage matériel et une transformation organique de la substance. » $\mathbf{9}$

La gymnastique visuelle et la tension psychique exigées par un voyage en train sont reconnues comme responsables de troubles nerveux qui affaiblissent l'organisme jusqu'au possible déclenchement d'une neurasthénie, maladie qui se traduit, entre autres symptômes, par une fatigue mentale et physique extrême10. Dans les années 1870-1880, le médecin physicien Georges Miller Beard institue la neurasthénie comme le trouble par excellence de la modernité occidentale, et plus spécifiquement de la société américaine jugée plus évoluée mais aussi plus sensible à certains maux, tel que le surmenage intellectuel accablant les élites du pays $\mathbf{1 1}$. Plus sensibles aux idéaux démocratiques, égalitaires et libertaires que la vieille Europe paralysée par le poids d'une histoire pluriséculaire et d'un système de classes rigide, les Etats-Unis permettraient, selon Beard, à chaque individu de développer ses ambitions avec une marge de manœuvre encourageant l'émergence de nouvelles idées, entreprises, inventions ou projets destinés non seulement à la prospérité nationale, mais aussi à la transformation radicale d'un monde au bénéfice du rayonnement américain. Cet immense champ d'action mis à disposition de tout individu - par définition intelligent et vigoureux - vivant sous ces latitudes, a cependant comme contrepartie de le soumettre à une plus grande fragilité physique et psychique
8 Wolfgang Schivelbusch, Histoire des voyages en train, Le Promeneur, Paris, 1990 [1977], pp. 60-61 (traduit par Jean-François Boutout).

9 "The Influence of Railway Travelling on Public Health ", in From the Lancet, Londres, 1862, p. 44, cité dans W. Schivelbusch, op. cit., pp. 61-62.

10 Voir Georges Frederick Drinka, The Birth of Neurosis: Myth, Malady and the Victorians, Simon and Schuster, New York, 1984; Anson, Rabinbach, The Human Motor: Energy, Fatigue and the Origins of Modernity, Basic Books, New York, 1990 (plus particulièrement le chapitre 6: "Mental Fatigue, Neurasthenia and Civilization", pp. 146-178).

11 Georges M. Beard, A Practical Treatise on Nervous Exhaustion (Neurasthenia): its Symptoms, Nature, Sequences, Treatment, The Classical of Psychiatry and Behavioral Sciences Library, New York, 1991 [1880]; Georges M. Beard, I/ nervosismo americano, S. Lapi, Castello, 1888 [1881] (traduit par Sofia Fortini Santarelli). 
"IMPRESSIONS ET COMPRESSIONS DE VOYAGE", DESSIN D'HONORÉ DAUMIER, TIRÉ DE LA SÉRIE LES CHEMINS DE FER (LES TRANSPORTS EN COMMUN), PARUE DANS LE CHARIVARI, 25 JUILLET 1843

12 Georges M. Beard, I/ nervosismo americano, op. cit., p. 99. Concernant le dernier critère, il pense en particulier aux femmes de la bonne société qui s'adonnent de manière immodérée à des activités mondaines et philanthropiques qui les rendent incapables d'accomplir leurs tâches maternelles et domestiques.

13/d., p. 109. II cite en particulier comme moyens de transport, les omnibus (p. 111) et le métro aérien de New York (p. 113).

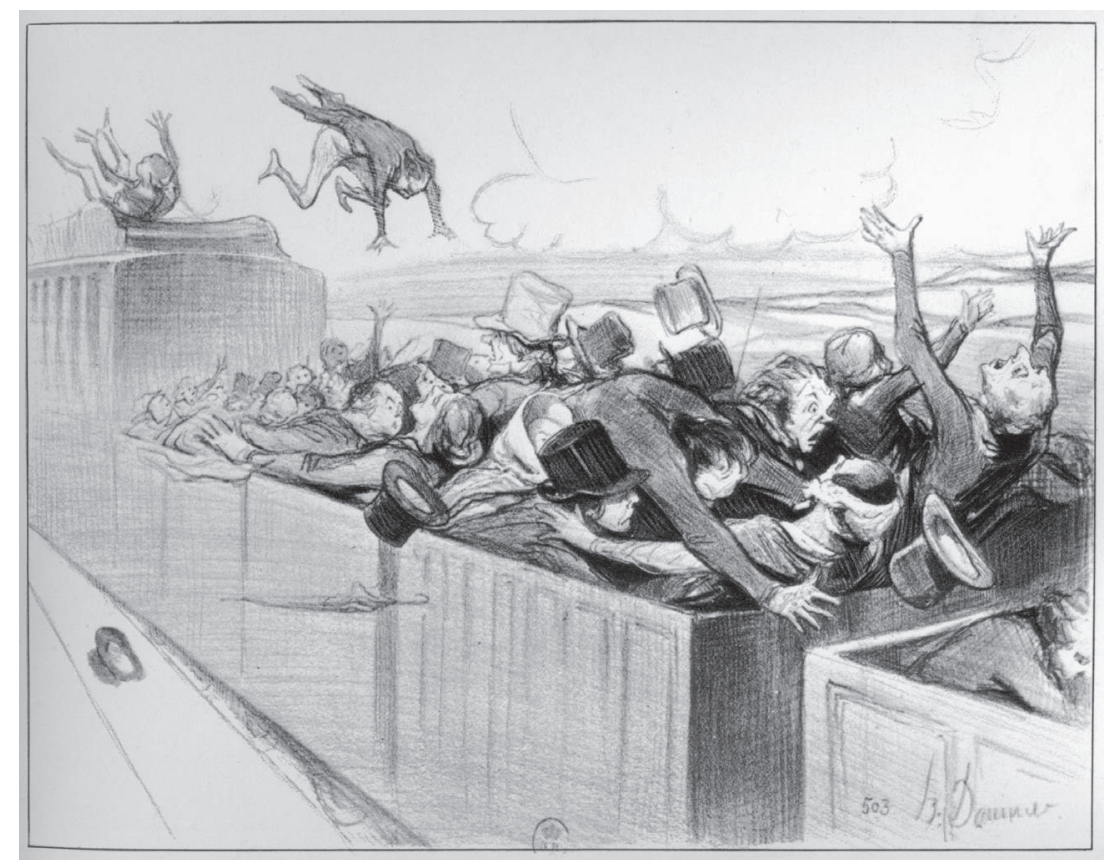

puisqu'il doit puiser ses forces dans un organisme qui peut rencontrer des limites face à un tel déploiement d'énergies créatrices. L'étiologie de la neurasthénie, selon Beard, est donc imprégnée d'un optimisme foncier fondé sur une confiance illimitée dans les ressources innées de l'homme civilisé, un optimisme qui tranche avec les théories médicales européennes plus enclines à intégrer des facteurs héréditaires dans le diagnostic des maladies nerveuses. Selon Beard, en effet, ce sont avant tout des causes environnementales et sociales qui déterminent la neurasthénie, pathologie qui atteint en priorité les classes moyennes et supérieures demeurant dans de grandes agglomérations et travaillant pour le bien commun. En tête de liste des origines possibles de la neurasthénie figure la "civilisation moderne" qui "consiste principalement dans ces cinq éléments - la vapeur, la presse écrite, le télégraphe, les sciences et l'activité mentale des femmes»12. Beard revient à plusieurs reprises sur le train comme générateur d'angoisses nouvelles - comme celle d'être en retard (problème lié à un besoin impératif de ponctualité) -, mais aussi d'incommodités telles que les "sons non rythmiques, non mélodiques et désagréables» produits dans «les manufactures, les moyens de transports, les voyages", et plus généralement «les grandes villes» qui sont bruyantes de nuit comme de jour ${ }^{\mathbf{1 3}}$. Il consacre surtout une section 
aux voyages en train considérés comme «la cause directe de maladies nerveuses» en raison du "dérangement moléculaire occasionné par de longs trajets", notamment chez les employés du chemin de fer $\mathbf{1 4}$. Le train peut aussi perturber l'équilibre mental des personnes qui développent une phobie du voyage en chemin de fer consécutivement à diverses péripéties, troubles visiblement très répandus à la lecture des nombreux témoignages et comptes rendus médicaux ${ }^{\mathbf{1 5}}$.

\section{Névrose traumatique et hystérie}

Si la neurasthénie en tant que nouvelle catégorie nosologique aura des répercussions importantes dans les cercles scientifiques de l'époque qui se réjouissent de pouvoir fournir aux patients démunis un label définitoire de leurs problèmes, les thèses de Beard connaîtront un accueil mitigé en fonction des pays et des cultures rencontrés $\mathbf{1 6}$. Alors qu'en Allemagne la "maladie de Beard» incorpore très vite le vocabulaire des neurologues et (un peu plus tard) des psychiatres réfléchissant aux répercussions de la modernité sur la santé $\mathbf{1 7}$, en France les médecins émettent des réserves à l'égard d'une théorie qui non seulement défie avec arrogance les savoirs scientifiques, culturels et technologiques européens, mais aussi évacue un peu trop rapidement la dimension congénitale de toute maladie mentale 18. Ces controverses sur l'origine exacte des névroses apparaissent avec clarté dans les débats concernant les désordres psychiques causés par les accidents de train, thème qui préoccupe très tôt les spécialistes $\mathbf{1 9}$ autant que l'imaginaire populaire fortement imprégné par les récits de grandes catastrophes ferroviaires 20 . Si les médecins anglo-saxons formulent très tôt des hypothèses sur ce qu'ils appellent le "Railway Spine »21, deux célèbres neurologues, Hermann Oppenheim à Berlin et Jean-Martin Charcot à Paris, s'affrontent sur le terrain de l'identification

EXPRESSION D'UN HOMME VICTIME DE NÉVROSE TRAUMATIQUE, D'APRĖS H. OPPENHEIM

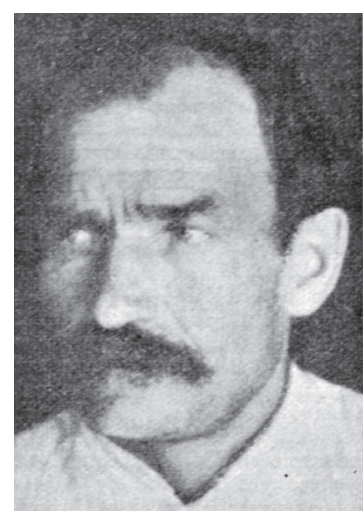

$14 / d .$, p. 115.

15 Je renvoie sur ce point à l'ouvrage très complet de W. Schivelbusch (op. cit.) qui traite cette question sur plusieurs chapitres.

16 Voir Marijke Gisjwijt-Hofstra et Roy Porter, Cultures of Neurasthenia from Beard to First World War, Rodopi, Amsterdam, 2001.

17 On trouve, parmi les promoteurs de la neurasthénie en Allemagne, Wilhelm Erb, Paul Julius Möbius ou Wilhelm Griesinger. Voir Joachim Radkau, Das Zeitalter der Nervosität: Deutschland zwischen Bismark und Hitler, C. Hanser, Munich, 1998.

18 Un très bon exemple de cette distance critique est donné par Maurice de Fleury dans son livre Les grands symptômes neurasthéniques: pathogénie et traitement, F. Alcan, Paris, 1910, pp. 281 et suivantes.

19 L'enjeu, pour les médecins, est de taille car ils sont mis à contribution à titre d'experts dans des procès qui opposent les victimes d'accidents aux compagnies ferroviaires. Ces rapports médico-légaux joueront un rôle significatif dans la construction scientifique et la reconnaissance juridique des maladies nerveuses.

20 Lire Marc Baroli, Lignes et lettres: anthologie littéraire du chemin de fer, Hachette Réalités, Paris, 1978.

21 Les chocs nerveux seront, dans un premier temps, référés "au désordre microscopique de la moelle épinière causé par l'ébranlement mécanique lié à l'accident", cette exégèse matérialiste étant, à la fin des années 1880 , périmée par une acception plus psychologique de la névrose traumatique. Schivelbusch, op. cit., p. 138. Les travaux de John Eric Erichsen (On Railway and Other Injuries of the Nervous System, 1866) et de Herbert Page (Injuries of the Spine and Spinal Cord and Nervous Shock, 1885) auraient servi de références. Voir Esther Fischer-Homberger, Die Traumatische Neurose: vom somatischen zum sozialen Leiden, $\mathrm{H}$. Huber, Bern/Stuttgart, 1975. 
22 Oppenheim remarque combien les temps modernes connaissent une recrudescence d'accidents liés aux dysfonctionnements divers de l'environnement urbain comme la chute des fils électriques de tramways sur les passants ou les désagréments sonores produits par les communications téléphoniques. Hermann Oppenheim, Lehrbuch der Nervenkrankenheiten: für Arzte und Studierende, S. Krager, Berlin, 1913, vol II, "Die traumatischen Neurosen (Unfallneurosen)", pp. 1537-1562.

23 Charcot explique que la névrose traumatique appartient à la "famille d'hystéries, distinctes nosographiquement les unes des autres en même temps qu'elles sont distinctes par la forme, [car] l'hystérie est une et indivisible". Selon lui, "qu'il s'agisse d'une collision de chemin de fer, d'un choc nerveux quelconque avec ou sans traumatisme, tremblement de terre, accident de voiture, ou au contraire du surmenage intellectuel ou génital [...]; peu importe, la névrose produite reste toujours essentiellement la même ", c'est-à-dire de type hystérique. Jean-Martin Charcot, Leçons sur l'hystérie virile, Le Sycomore, Paris, 1984 [1885-1891], pp. 178179. Sur l'hystérie, lire Mark S. Micale, Approaching Hysteria. Disease and its Interpretations, Princeton University Press, Princeton-New Jersey, 1995; Georges Didi-Huberman, Invention de l'hystérie. Charcot et l'iconographie de la Salpêtrière, Macula Scènes, Paris, 1982.

DESSIN ANGLAIS (1831) RAILLANT CERTAINS INCONVÉNIENTS DU VOYAGE EN TRAIN exacte d'une pathologie reconnue par les deux savants comme due principalement à un choc extérieur plus ou moins violent (accident de train ou de travail, chute, incendie, secousses électriques, etc.) ou une très forte émotion psychologique entraînant une série de symptômes tels qu'insomnie, fatigue, maux de tête, désorganisation et hyperexcitabilité sensorielles, paralysie, angoisses, phobies, etc.

Alors qu'Oppenheim et ses collaborateurs proposent le terme de névrose traumatique pour désigner la pathologie touchant les personnes ayant survécu à une collision ferroviaire sans blessures apparentes $\mathbf{2 2}$, Charcot préfère quant à lui inclure ces cas particuliers dans la famille de l'hystérie qui, selon lui, subsume toutes les déclinaisons possibles de névroses, notamment l'hystéro-neurasthénie ${ }^{23}$. Cette construction sémantique utilisée pour qualifier une maladie qui terrasse en majorité des hommes dans la force de l'âge va susciter de franches oppositions au sein des cénacles scientifiques soucieux de ne pas féminiser davantage des troubles plongeant les personnes dans un état de vulnérabilité habituellement imputé à la nature féminine. Or, Charcot démontre, contrairement à l'opinion commune, que loin d'être une aberration lexicale - à condition de la débarrasser de son étymologie première (utérus en grec) -, l'hystérie concerne autant les hommes que les femmes, ainsi que toute la pyramide sociale, et plus particulièrement les classes laborieuses considérées comme plus robustes que les couches

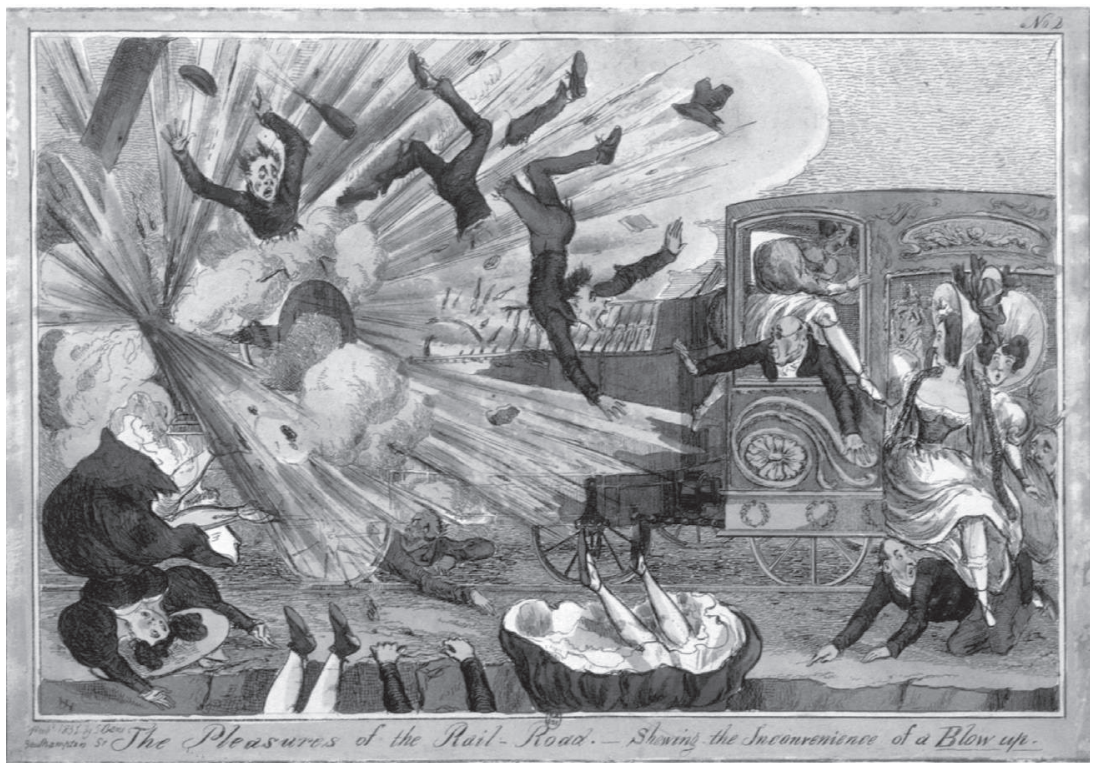




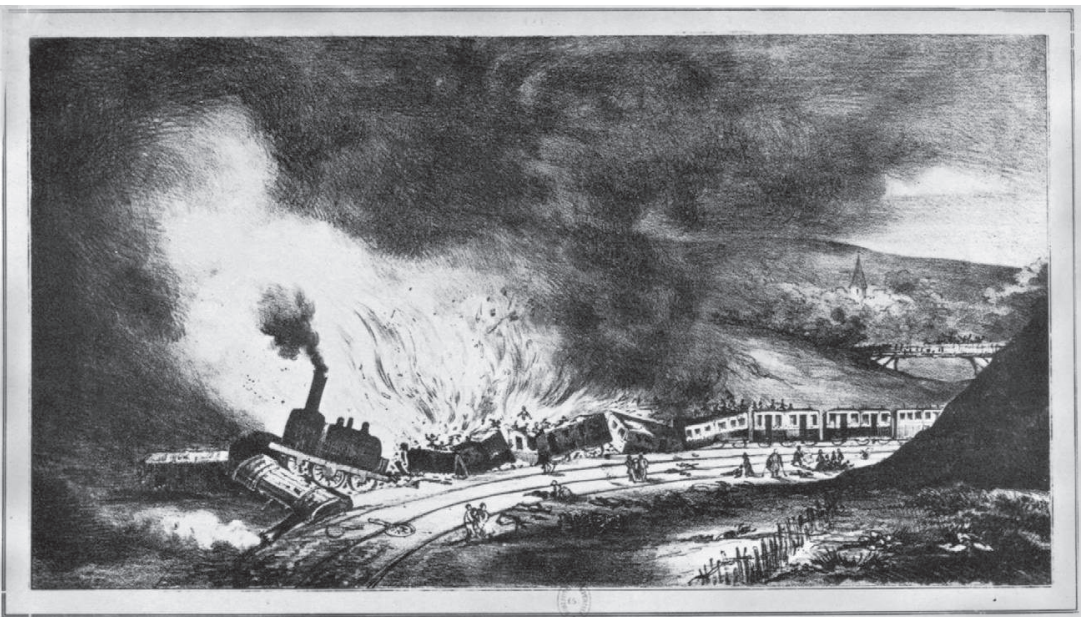

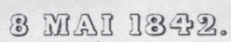

éduquées, mais aussi plus sujettes aux heurts de la vie moderne. Dans ses leçons sur l'hystérie virile destinées à ses étudiants, Charcot expose un nombre important d'histoires morbides liées à des accidents de chemin de fer, d'omnibus ou de tramway qui fonctionnent comme facteur déclenchant, alors qu'une prédisposition héréditaire vient compléter le tableau pathogène, les professions ferroviaires (chauffeur de locomotives, garde-freins, etc.) étant surreprésentées $\mathbf{2 4}$.

Ces discussions, loin de se réduire à des querelles d'initiés, doivent être replacées sur le fond d'un contexte socio-historique et idéologique européen empreint d'inquiétudes sur l'affaiblissement généralisé du corps social composé d'individus dont les performances physiques et mentales font l'objet de nombreuses études dans le champ scientifique $\mathbf{2 5}$. Solidaires d'un projet éducatif et hygiéniste visant à lutter contre la dégénération de la race humaine occidentale, les milieux médicaux vont prendre en charge un discours sur la fatigue mentale et le surmenage intellectuel qui menacent chaque nation, et notamment leurs jeunes générations 26 . En France, l'étiologie des maladies nerveuses opère ainsi une synthèse entre les thèses d'Augustin Morel sur le déclin bio-physiologique de l'être humain civilisé27, celles de Lamarck sur l'hérédité des caractères acquis $\mathbf{2 8}$ et la vision alarmiste des séquelles laissées par une société industrielle surdéveloppée ${ }^{29}$. Malgré la prégnance d'une vision qui accorde à l'asthénie congénitale une place décisive, les thérapeutes, comme Alexandre Cullerre, enregistrent l'importance des effets surstimulants de la modernité en observant que le XIX siècle "est un siècle
PREMIÈRE GRANDE CATASTROPHE FERROVIAIRE, MEUDON

24 Jean-Martin Charcot, Leçons sur l'hystérie virile, op. cit. Sa leçon introductive démontre l'ampleur de l'enjeu puisqu'il s'agit de battre en brèche une série de préjugés sociaux et idéologiques qui sous-tendent l'étiologie traditionnelle de l'hystérie: "L'hystérie mâle n'est donc pas, tant s'en faut, très rare. Eh bien! messieurs, si j'en juge d'après ce que je vois chaque jour parmi nous, ces cas-là sont bien souvent méconnus, même par des médecins très distingués. On concède qu'un jeune homme efféminé puisse après des excès, des chagrins, des émotions profondes, présenter quelques phénomènes hystériformes; mais qu'un artisan vigoureux, solide, non énervé par la culture, un chauffeur de locomotive par exemple, nullement émotif auparavant, du moins en apparence, puisse, à la suite d'un accident de train, d'une collision, d'un déraillement, devenir hystérique, au même titre qu'une femme, voilà, paraît-il, qui dépasse l'imagination. Rien n'est mieux prouvé, cependant, et c'est une idée à laquelle il faudra se faire" (p. 37). A ce propos, lire l'introduction aux Leçons sur l'hystérie virile de Michèle Ouerd, pp. 11-31.

25 Voir Angelo Mosso, La fatigue intellectuelle et physique, F. Alcan, Paris, 1908 [1891]. Sur les liens entre physiologie et éducation physique, lire Christian Pociello, La science en mouvements. Etienne Marey et Georges Demenÿ (1870-1920), PUF, Paris, 1999.

26 Voir Rabinbach, The Human Motor, op. cit.

27 Bénédict-Augustin Morel, Traité de dégénérescences physiques, intellectuelles et morales de l'espèce humaine et de ses causes qui produisent ces variétés maladives, Baillière, Paris, 1857.

28 Jean-Baptiste Lamarck, Philosophie zoologique, ou exposition des considérations relatives à l'histoire naturelle des animaux, Savy, Paris, 1873 [1809].

29 Lire Christopher E. Forth, "Neurasthenia and Manhood in fin-de-siècle France", Cultures of Neurasthenia from Beard to First World War, op. cit., pp. 329-361. 
30 Alexandre Cullerre, Nervosisme et névroses: Hygiène des énervés et des névropathes, Baillière, Paris, 1887, p. 12.

31 Fernand Levillain, Essais de neurologie clinique. Neurasthénie de Beard et états neurasthéniformes, Maloine, Paris, 1896, p. 67, p. 31, p. 202, p. 220 (il emploie le terme "théâtrophobie") et p. 8.

32 Pour un rapport complet d'un cas de névrose traumatique provoquée par un déraillement de chemin de fer, lire le cas CVII, id., pp. 259-269. II s'agit pour Levillain d'attester auprès des instances juridiques compétentes du caractère soudain et inédit de symptômes imputables à l'accident. II résume par ailleurs les positions des différentes écoles sur la névrose de chemin de fer en revenant sur la question de la terminologie à emprunter pour dresser le pronostic.

33 François Foveau de Courmelles, Comment on se défend contre la neurasthénie: la lutte contre le surmenage mental, L'Edition médicale française, Paris, 1900, p. 14.

34 J'emprunte cette expression à Ben Singer, "Modernity, Hyperstimulus, and the Rise of Popular Sensationnalism ", dans Leo Charney et Vanessa R. Schwartz (éd.), Cinema and the Invention of Modern Life, op. cit., pp. 72-99.

35 Sabine Hake souligne la récurrence des métaphores martiales employées pour évoquer les effets néfastes des images cinématographiques sur le spectateur qui se voit assailli, bombardé, attaqué par des rayons ou des éclats qui lui explosent à la figure. Sabine Hake, The Cinema's Third Machine. Writing on Film in Germany, 1907-1933, University of Nebraska Press, Lincoln et Londres, 1993, p. 30. de mouvement accéléré. Nous sommes dans une perpétuelle effervescence; nos systèmes nerveux restent dans un état de tension qui ne se relâche jamais» $\mathbf{3 0}$. Pour sa part, le médecin Fernand Levillain, ancien élève de Charcot, reconnaît que la névrose traumatique «est peut-être la seule névrose qui puisse se développer accidentellement de toute pièce sans hérédité préalable» donnant à l'appui une série d'exemples tirés de sa pratique clinique, comme une pathologie occasionnée suite à une "rencontre de tramway", une phobie du voyage en train, mais aussi des tunnels, de la foule, du théâtre et des bals chez des personnes dont «l'hyperexcitabilité sensorielle et morale»31, peut être exacerbée par la lecture intensive de la presse à sensations relatant faits divers et incidents tragiques 32. Pour le docteur François Foveau de Courmelles, le sujet neurasthénique n'est plus en mesure de faire face aux rythmes trépidants du climat urbain où «la foule, le mouvement, un bruit, un geste ou une broutille [lui] sont insupportables» $\mathbf{3 3}$. Ainsi, on constate combien les pathologies neurologiques s'enracinent dans les chocs culturels d'une modernité qui agit comme un réservoir de menaces potentielles étayées sur le modèle de la collision qu'emblématisent parfaitement le train, sa mécanique de déplacement et ses déraillements possibles.

\section{Le cinéma entre choc (électrique) et ennui (hypnotique)}

Si le train est reconnu dès son invention comme coupable d'engendrer des tensions et des chocs nerveux irréversibles pour la santé publique, le cinéma comme agent de construction d'une «modernité neurologique» $\mathbf{3 4}$ deviendra également la cible de recherches sur la nocivité des images en mouvement pour les nerfs et les yeux. La fréquentation régulière des salles obscures constitue, selon les mouvements de réforme du cinéma émergeant durant les deux premières décennies du $\mathrm{XX}^{\mathrm{e}}$ siècle, une menace pour le bien-être de l'homme, à l'égal de l'environnement urbain qui assaille le sujet percevant d'impressions perturbant à la longue certaines fonctions vitales. En effet, après 1900, les effets de la modernité continuent d'être ressentis en termes d'aliénation, de pression et d'attaque sur les psychismes continuellement sollicités sur le plan sensoriel au point d'être déréglés durablement ${ }^{35}$. En Allemagne, divers groupes d'éducateurs, de psychologues et de criminologues qui s'emploient à associer étroitement l'accroissement des maladies nerveuses (et des comportements déviants) aux phénomènes propres à l'urbanité, mènent notamment des enquêtes sur les réactions physiologiques induites par la vision d'un film projeté sur un écran très lumineux, dans une salle sombre, mal aérée, inconfortable et propice aux actes licencieux encouragés par la promiscuité sociale. Le réformateur Nado Felke, par exemple, 


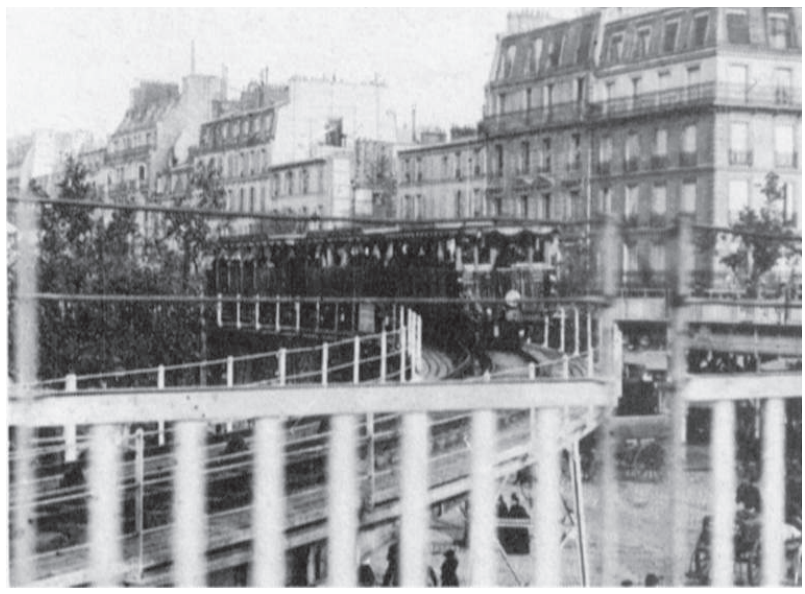

estime que le psychisme doit se plier à des efforts contre nature afin de faire la synthèse d'une multitude d'images défilant à grande vitesse ${ }^{36}$. Rappelant les commentaires sur les nuisances du voyage en train, cet argument participe avant tout d'une stratégie qui vise à modérer l'assiduité des jeunes fréquentant régulièrement les cinémas. Si ces derniers reconnaissent d'ailleurs que le film peut occasionner des désagréments tels que l'inflammation des yeux ou les maux de tête, leur goût pour les stimulations visuelles et émotionnelles l'emporte largement ${ }^{37}$. Comme l'a démontré l'étude de Sabine Hake, dès les débuts de son histoire, le cinéma a été stigmatisé pour causer bien des dommages, allant des bouleversements perceptifs et nerveux à la cécité, la pneumonie, la folie ou les maladies vénériennes ${ }^{38}$. Il n'est pas indifférent de noter que les reproches adressés au cinéma recoupent ceux qui ont été soulevés bien avant son invention à propos du chemin de fer, également ressenti comme un espace mettant en danger l'intégrité physique et mentale de ses usagers via une image à la fois fuyante et trépidante qui dépasse les compétences oculaires humaines.

En 1917, dans un article consacré aux méfaits du cinéma sur les enfants, le psychologue Hugo Münsterberg évoque le risque d'une irritation dangereuse des nerfs et des yeux chez les jeunes spectateurs facilement impressionnables et structurellement plus délicats que les adultes ${ }^{39}$. De plus, dans sa théorie psychophysiologique du cinéma datant de la même année, il relève que la surstimulation sensorielle peut compromettre davantage la santé des personnes neurasthéniques, alors que le contenu des images porte en lui le germe d'une possible «infection psychique» chez des sujets facilement suggestionables $\mathbf{4 0}$. Or, dans
PHOTOGRAMME D'UN FILM LUMIĖRE, PLATE-FORME MOBILE ET TRAIN ÉLECTRIQUE, EXPOSITION UNIVERSELLE DE PARIS 1900. À NOTER L'INSCRIPTION DANS L'IMAGE D'UN DOUBLE MOUVEMENT, AINSI QUE LE RAPPORT ENTRE LES PREMIER ET SECOND PLANS RAPPELANT LE MODĖLE DE LA VISION EN TRAIN
36 Nado Felke, "Die Gesundheitsschädlichkeit des Kinos", Die Umschau, vol. 17, n 3, 1913, p. 254, cité dans Michael Cowan, "Theater and Cinema in the 'Age of Nervousness : Der Andere by Paul Lindau (1894) and Max Mack (1913)", in Cinema \& Cie, $n^{\circ} 5$, Fall 2004, p. 74.

37 Franz X. Schönhuber, Das Kinoproblem im Lichte von Schülerantworten, Leipzig, A. Hasse, 1918, cité dans Sabine Hake, The Cinema's Third Machine, op. cit., p. 49.

$38 \mathrm{lbid}$. A noter que ces mêmes maux apparaissent aussi dans les débats sur le chemin de fer. Arago, par exemple, mentionne dans un discours à la Chambre des députés en juin 1836, qu'en traversant les tunnels, "les personnes sujettes à la transpiration seront incommodées, qu'elles gagneront des fluxions de poitrines, des pleurésies, des catarrhes". Cité dans Marc Baroli, Lignes et lettres, op. cit., p. 67. II faudrait ajouter à cette liste, le crime et le vol qui trouvent dans la salle de cinéma, comme dans le compartiment de train, un champ d'application particulièrement aisé, notamment en raison de la couverture sonore fournie soit par les bruits de machines, soit par l'accompagnement musical et verbal.

39 Hugo Münsterberg, "Perils to Childhood in the Movies ", in Mother's Magazine, $\mathrm{n}^{\circ} 12$, 12 février 1917, dans The Photoplay: A Psychological Study and Other Writings (Allan Langdale, éd.), Routledge, Londres, 2002, p. 195.

40 Hugo Münsterberg, The Photoplay, op. cit., p. 154. 
41 ld., p. 145.

42 Emilie Altenloh, Zur Soziologie des Kinos, Diedrichs, Jena, 1914, p. 56. Citée dans Sabine Hake, The Cinema's Third Machine, op. cit., p. 46.

43 Reconduisant les préjugés de sa classe et de son temps, Altenloh ancre dans la nature féminine ce besoin d'évasion du quotidien - par ailleurs proportionnel au statut social pour ensuite le rattacher à un besoin de consommation assouvi dans les magasins de luxe qui proposent des produits indirectement vantés dans les films à l'affiche. Ainsi, la femme fréquenterait le cinéma par frivolité, alors que les hommes seraient mus par des raisons plus "sérieuses". Emilie Altenloh, Zur Soziologie des Kinos, op. cit., p. 78. Cité dans Sabine Hake, The Cinema's Third Machine, id., p. 78.

44 Fernand Levillain commente qu'à un patient ayant développé une série de phobies à la suite de divers chocs psychologiques, "on conseilla la distraction: il vint à Paris, mais n'y put trouver aucun plaisir; le bruit des rues, le mouvement de la foule l'assourdissaient". Levillain, Essais de neurologie clinique, op. cit., p. 46.

45 Bien des médecins mettent sur le compte d'une débauche de plaisirs tels que les sorties au théâtre, au café-concert, au cabaret, mais aussi la lecture de romans (particulièrement réalistes et naturalistes) l'épuisement moral et intellectuel de leurs patients. Les thèses d'Adrien Proust, père du célèbre écrivain, sont particulièrement édifiantes en la matière puisque le médecin aurait pris comme modèle son propre fils pour dresser le profil type du neurasthénique doté à la fois d'une sensibilité extrême aux stimuli extérieurs et d'une faiblesse intérieure qui l'empêche de maintenir le contrôle de ses émotions. Voir Adrien Proust et Gilbert Ballet, L'hygiène du neurasthénique, Masson et Cie, Paris, 1897. un autre passage, il met aussi en évidence les répercussions positives que peut avoir, par exemple, l'accompagnement musical sur le visionnement de films traînant en longueur, car il favoriserait à la fois le relâchement des tensions internes et le maintien de l'attention 41. Münsterberg reconnaît donc implicitement la dualité d'un spectacle aux conséquences contrastées, même s'il se concentre principalement sur ses fonctions morales et pédagogiques.

Optant pour une approche sociologique, l'élève d'Alfred Weber, Emilie Altenloh établit dans sa thèse de doctorat une forte connexion entre le cinéma, la modernité, la nervosité et l'ennui, ce dernier résultant précisément d'un mode de vie saturé d'impressions sensorielles et de phénomènes éphémères:

«Le cinéma et son public sont des produits typiques de notre temps qui se caractérise par des activités constantes et une agitation nerveuse. Enfermés durant toute la journée dans leurs lieux de travail, les gens ne peuvent s'empêcher de courir se détendre. En passant devant un cinéma, ils y entrent afin de chercher, pour un court instant, une diversion et une distraction, même s'ils sont déjà préoccupés de savoir comment ils rempliront les prochaines heures. $\mathbf{4 2}$

Si le cinéma devient un espace de délassement pour le spectateur type de l'époque - un homme jeune, ouvrier d'usine, peu payé mais avide d'activités récréatives -, il attire également des femmes qui, selon Emilie Altenloh, se rendent au cinéma de manière parfois compulsive pour compenser le vide et la monotonie de leur existence 43 . En attribuant au cinéma une vocation à la fois stimulante et relaxante, Altenloh ne parvient pas vraiment à faire le partage entre le spectateur harassé en quête de rémission et celui qui trompe son ennui au travers d'une évasion sans cesse recommencée, allant jusqu'au développement d'une dépendance. Chez Münsterberg comme chez Altenloh, on découvre combien les vertus "thérapeutiques» du cinéma peuvent se combiner avec leurs opposés pour fonctionner sur un mode de complémentarité qui explique l'intérêt pour un médium qui à la fois envoûte (sur le mode de l'hypnose) et inquiète (sur le mode de l'excitabilité pathogène). Dans le discours médical de la névrose, cette pensée disjonctive - partagée sur les bienfaits ou malfaisances de la modernité - apparaît également lorsque les thérapeutes conseillent à leurs patients hystéro-neurasthéniques d'opter tantôt pour la distraction en s'immergeant dans un bain de foule ou en goûtant les plaisirs urbains $\mathbf{4 4}^{\mathbf{4}}$, tantôt pour le repos absolu hors des grandes agglomérations, au bord de la mer, à la campagne ou dans un sanatorium $\mathbf{4 5}$. Beard, qui aime à comparer le fonctionnement du système nerveux à celui d'une machine électrique, explique que les 


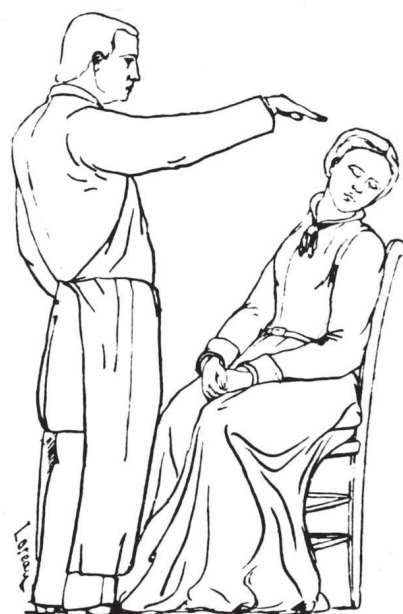

"SCHÉMA DE PASSE HYPNOTIQUE", ICONOGRAPHIE PHOTOGRAPHIQUE DE LA SALPÊTRIÈRE,

D.-M. BOURNEVILLE ET P. RÉGNARD, VOL. III, 1879-1880

pertes d'énergies nerveuses peuvent provenir autant d'une surcharge de tensions que d'un appauvrissement d'apport électromagnétique à l'organisme 46. Identifié à une force vitale circulant dans les multiples ramifications et connexions du système nerveux, le tonus mental et physique doit, selon lui, atteindre un juste équilibre pour que la "machine animale» $\mathbf{4 7}$ ne tombe pas en panne. Inspirée par l'invention de la lampe électrique à incandescence de Thomas Edison 48, la métaphore électro-physiologique employée par le médecin américain permet de tirer deux conclusions préliminaires. Elle prouve, d'une part, combien la pensée du corps médical est contaminée de toutes parts par cette modernité qui fonctionne à la fois comme cause et effet d'une maladie protéiforme dont la systématisation scientifique vise à maîtriser des phénomènes qui, pourtant, ne cessent de l'excéder. D’autre part, elle va justifier le recours à l'électrothérapie comme remède aux divers dysfonctionnements neurologiques traités par le biais de courants localisés ou généralisés qui agissent comme des «frictions» tantôt excitatives, tantôt décontractantes 4 . Dans cette perspective, l'électricité pourrait bien avoir servi de paradigme aux différentes pratiques discursives qui tentent de saisir la spécificité du sujet moderne.

Les dispositifs ferroviaire et cinématographique vont précisément constituer des sortes de "laboratoires» permettant d'éprouver les thèses marquées par le sceau de la modernité, cristallisant ainsi autant les peurs que les espoirs liés à la transformation d'un monde auquel, comme le note Ben Singer, il faudra du temps pour s'habituer 50. Les moyens de défense déployés face à cette "hydre» aux effets multiples et contradictoires ont maintes fois été théorisés, comme le démontrent
46 A propos de la diminution de la force nerveuse, il établit la chose suivante: "Quand dans le circuit s'interposent de nouvelles fonctions, comme la civilisation moderne, [...] il se peut que la quantité de force soit insuffisante pour maintenir allumée toutes les lampes; celles qui sont plus faibles s'éteignent immédiatement, ou, comme il arrive plus fréquemment, qu'elles brillent faiblement; elles ne s'épuisent pas mais elles produisent une lumière insuffisante et incertaine. Telle est la philosophie de la nervosité moderne". Beard, II nervosismo americano, op. cit., p. 102.

47 Dans ce contexte, l'expression du physiologiste Etienne-Jules Marey convient à merveille. Voir La machine animale, Baillière, Paris, 1873.

48 L'intérêt soutenu de Beard pour l'électricité le mènera à collaborer avec Edison dans les années 1870. Suite à des expériences communes sur des étincelles produites par un générateur, il publiera un article, "The Alleged New Force", dans lequel il pense avoir trouvé l'origine de la force vitale. Voir Drinka, The Birth of Neurosis, op. cit., p. 191.

49 Tous les manuels de neurologie contiennent des chapitres sur les méthodes électrothérapeutiques, que ce soit la galvanisation et la faradisation (usitées en Autriche et en Allemagne), ou la franklinisation et les bains électrostatiques (plus fréquents en France). Voir Fernand Levillain, Essais de neurologie clinique, op. cit., p. 410.

50 L'ouvrage de Ben Singer propose précisément d'expliquer cette attitude ambivalente à l'égard d'une modernité qui à la fois fascine et fait horreur, et ceci à travers une analyse des spectacles à vocation sensationnaliste, et plus précisément du genre du mélodrame. Ben Singer, Melodrama and Modernity. Early Sensational Cinema and Its Contexts, Columbia University Press, New York, 2001. 
51 Georg Simmel, "The Metropolis and Mental Life " [1903], The Sociology of Georg Simmel, op. cit., pp. 409-424.

52 Walter Benjamin, "Sur quelques thèmes baudelairiens" [1939], CEuvres III, Paris, Gallimard, Folio/Essais, 2000, pp. 329-390; Sigmund Freud, "Au-delà du principe de plaisir" [1920], Essais de psychanalyse, Paris, Payot, 1997, pp. 40-115.

53 Sur les notions de choc et d'ennui, lire Patrice Petro, "After Shock/Between Boredom and History", dans Patrice Petro (ed.), Fugitive Images. From Photography to Video, Indiana University Press, Bloomington-Indianapolis, 1995, pp. 265-284.

54 Voir par exemple, Hippolyte Bernheim, Hypnotisme, suggestion, psychothérapie, avec considérations nouvelles sur l'hystérie, Fayard, Paris, 1995 [1903]. les propositions de Georg Simmel - avec sa notion d'«attitude blasée» caractérisant le citadin $\mathbf{5 1}$ - ou de Walter Benjamin - avec sa relecture de Poe et de Baudelaire au travers du Freud des années 1920 et de son concept de pare-excitations $\mathbf{5 2}$. La distraction, toutefois, n'est pas la seule échappatoire qui s'ouvre au sujet moderne conditionné par son biotope, puisqu'au sein des trains et des cinémas se sont développées d'autres tactiques de résistance aux surstimulations sensorielles comme la somnolence, l'apathie, le sommeil et l'ennui ${ }^{53}$, conditions parfois connues du sujet névrosé. Parallèlement, une partie de la communauté médicale, et particulièrement de la psychiatrie, découvre les ressources de l'hypnose qui, à défaut d'efficience thérapeutique véritable, devient un instrument herméneutique proposant une alternative au paradigme électro-physiologique qui domine la neurologie54. Cet état hypnotique
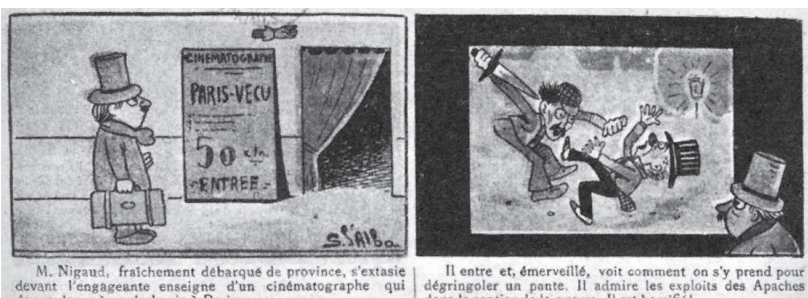

Il entre et, émerveillt, voit comment on s'y prend pour
degringoler un pante. II adimire les exploits des Apaches devant l'engageante enseigne d'u
donne des scines de la vie a Paris.
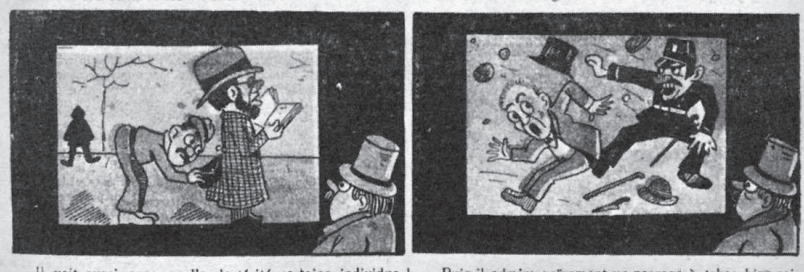

1t. voit aussi avec quelle dextérite certa
allegent la poche de leurs contemporains.

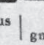

Puis il admire naîvement un passage à tabac bien soi-
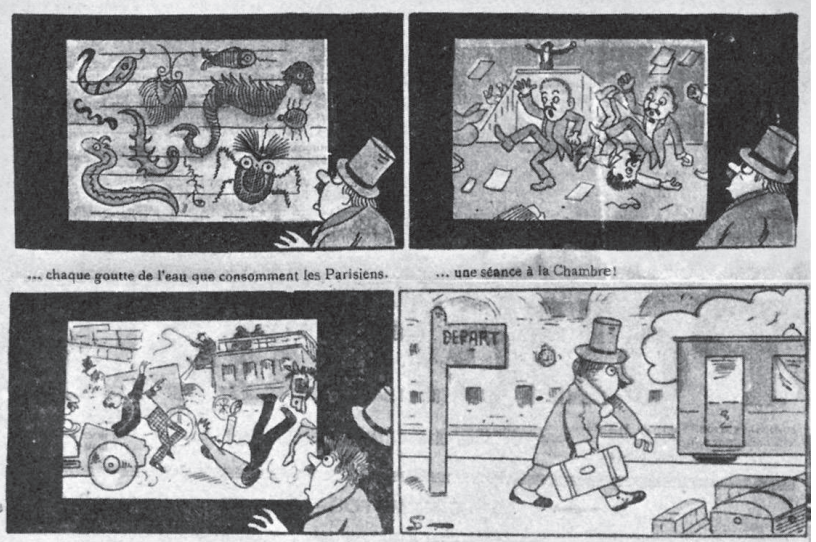

POLICHINELLE, 13 JANVIER 1907. REPRÉSENTATION DES DANGERS À LA FOIS DE LA VILLE ET DU CINÉMA POUR LE NON INITIÉ

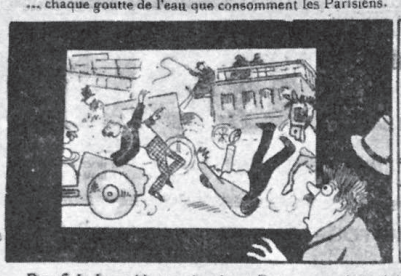

Pour finir, les accidents qui arrivent (fort souvent, hellast) I Alors M. Nigand, degoate de Paris, prend sa valise et,
par le premier train, regagae ses pénates, 


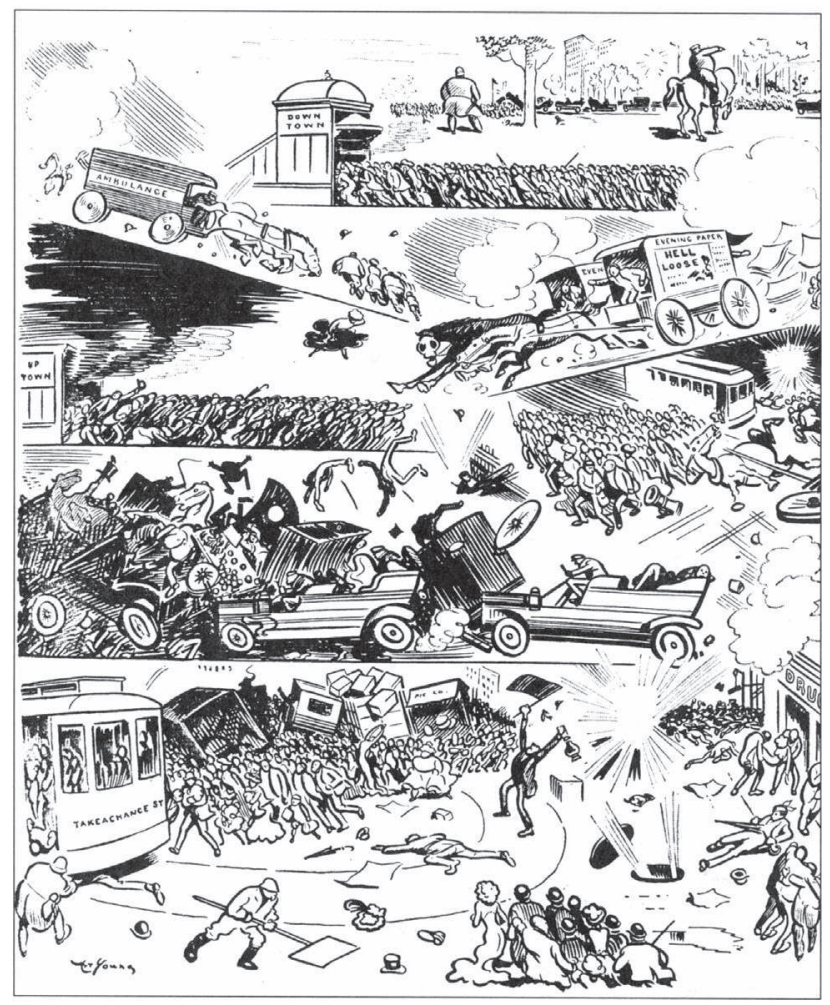

peut aussi à l'occasion gagner le voyageur de train, comme le spectateur de cinéma, s'ils choisissent d'abaisser leur seuil de vigilance coutumier afin de naviguer entre la veille et le sommeil, et de s'abandonner à la captation imaginaire. Pris dans un mouvement d'oscillation constant, à l'instar du pendule usité par l'hypnotiseur, le sujet moderne peut facilement basculer de l'hystérie à l'hypnose, du choc à l'ennui, de l'apathie à la versatilité, de la réactivité à la suggestibilité. Les discours accueillant les arrivées certes différées, mais homothétiques, du train et du cinéma, semblent emprunter les mêmes cheminements le long de cette ligne qui relie le pôle de l'hystérie au pôle de l'hypnose, tensions permanentes dont le va-et-vient et le balancement incessants du voyage en train - mais aussi de la pensée et du désir, essentiels au cinéma 55 - pourraient être les symboles.
"NEW YORK CITY: IS IT WORTH IT?",

MAI 1909. ILLUSTRATION DE PRESSE

METTANT EN SCĖNE DE MANIÈRE SÉQUENTIELLE

LES PÉRILS DE LA GRANDE VILLE $\mathbf{5 5}$ Sylvie Nysenbaum, "Une pensée qui va et vient", in Nouvelle Revue de Psychanalyse, $n^{\circ} 25$, printemps 1982, pp. 229-252. L'auteure analyse l'emprise du mouvement de va-etvient sur la théorie freudienne du psychisme, en rappelant notamment l'importance du motif de balancement du train comme porteur d'excitation sexuelle, à l'instar d'autres figures rythmiques langagières ou mentales. 